\title{
Analysis of mechanical characteristics force signal of high voltage circuit breaker
}

\author{
Yang Gao ${ }^{1, a^{*}}$, Zhang Bo ${ }^{1,}$, Aoran $\mathrm{Xu}^{1,}$, , Fangxin Dai ${ }^{2, b}$, Weiliang Zheng ${ }^{2, b}$ \\ ${ }^{1}$ Shenyang Institute of Engineering, Shenyang City, Liaoning Province, China \\ ${ }^{2}$ Yingkou power supply company, Liaoning Province, China State Grid Corp \\ aemail: gaoyangsie@163.com
}

\begin{abstract}
Key words : Characteristic point; Wavelet packet analysis; Action time; Tension force signal
\end{abstract}
\begin{abstract}
According to the characteristics of the corresponding rigid points in the actual force signal, the background and characteristics of signal analysis are described in this paper for the purpose of obtaining this feature point and the extraction and calculation of the moment of the force signal is realized, and the wavelet packet analysis is chosen for the analysis of the force signal of the tierod and the feature extraction. Finally, the realization process of the wavelet packet analysis of force signal is described in detail.The action time of force measurement and that of the main circuit of the same action process are analyzed to demonstrate the correctness of the method according to the test results of the test system.
\end{abstract}

\section{Introduction}

In order to obtain the just minutes from the force signal, this paper proposed a signal processing scheme of wavelet packet decomposition and reconstruction and realized the extraction and calculation of the moment of the force signal. Through the comparison of the results of the force signal processing and the results of the traditional off-line measurement, the results showed that, the error of the just moment of the circuit breaker based on the force sensor signals less than $0.2 \mathrm{~ms}$, thus this paper further demonstrated the feasibility of on-line measurement of mechanical characteristics of circuit breaker with force signal.

\section{Background of signal analysis}

Circuit breaker is working in high voltage environment, and there is a lot of electromagnetic interference, at the same time, because of the complex structure of the circuit breaker, there will be a variety of environmental noise in the on-line measurement. According to the test system actual curve, there are a lot of the burr pull in the force curve during the process of opening and closing, at the same time, it is a singular point of the curve. This requiring effective method to distinguish noise and useful signal, and extracting the feature time point of the corresponding sub switch from the useful signal, the basic idea of Fu Liye transform is decomposing the signal into a number of different frequencies sine wave superposition, it decomposes the signal into the whole frequency range, so losing the time information, and it can't be used to analyze the information in the frequency domain information or a certain frequency corresponding time information, which can't be used to analyze the time information at a certain time. It is a kind of frequency localization signal analysis method with window size and shape variable, it has with high frequency resolution and low 
time resolution in the low frequency part, and has high time resolution and low frequency resolution in the high frequency part, So you can see the picture signal, and the details of the signal. The real wavelet analysis is the multi resolution analysis, and its realization process is shown in figure 1.

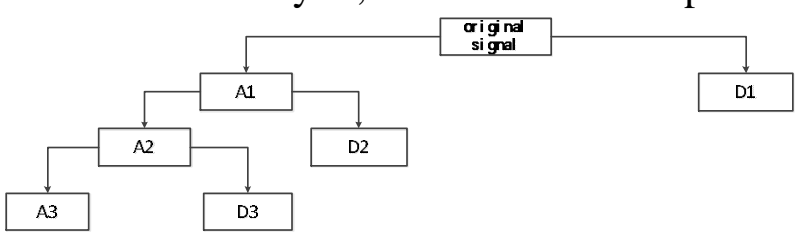

Fig.1 Schematic diagram of multi resolution analysis

Wavelet packet analyzing make up for the above analysis of the high frequency signal insufficient capacity, it's implementation process as shown in figure 2.

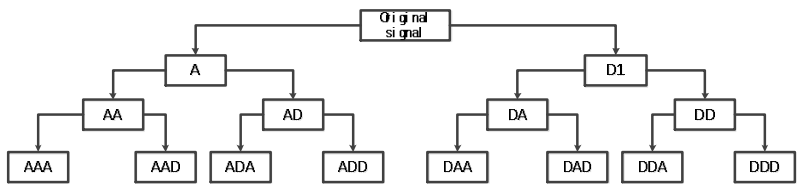

Fig. 2 Schematic diagram of wavelet packet decomposition

Wavelet packet making the further decomposition to multi resolution analysis without subdivision of the high frequency part, more accurate analysis to the signal, improving the time-frequency resolution, and basing on the analyzed characteristics of the signal, adaptively select the appropriate frequency band to adapt the spectrum of the signal. During the process of switching opening and closing, tension force signal is a non-stationary signal in the presence of interference, first moment of the switching opening and closing corresponding singular points on the corresponding signal. This paper using the wavelet packet analysis and extract singular point at the first action moment in the tension force signal.

\section{Wavelet packet analysis of switching force signal}

The signal is decomposed and reconstructed by wavelet packet, and the singular point of signal and the extremum of the high frequency coefficient of one layer are corresponding. There are two key issues in the wavelet packet decomposition and reconstruction of signals: the choice of wavelet bases and decomposition level. In view of the fact that there are plenty of analysis tools about wavelet packet in MATLAB, the paper takes the strategy that: firstly, according to the empirical data set a common decomposition level; secondly, using the common wavelet packet in MATLAB decompose and reconstruct pull curves in opening and closing. Using common wavelet bases analyze pull force signal one by one by the arithmetic to determine the optimal wavelet basis used to analyze force signal and corresponding optimal decomposition level.

The 23 specific types of wavelet used in Matlab are shown in table 1.

Table 1 common wavelets in Matlab

\begin{tabular}{|c|c|c|c|c|}
\hline Haar & Db2 & Db3 & Db4 & Db5 \\
\hline Db6 & Db7 & Db8 & Db9 & Db10 \\
\hline Bior1.1 & Bior2.2 & Bior2.6 & Bior3.5 & Bior3.9 \\
\hline Bior6.8 & Coif1 & Coif2 & Coif3 & Coif4 \\
\hline Sym3 & Sym4 & Sym5 & Sym6 & \\
\hline
\end{tabular}

According to the actual curve of the experiment, and take A phase as an example, the closing pr ocess of the force signal is as figure 3 . 


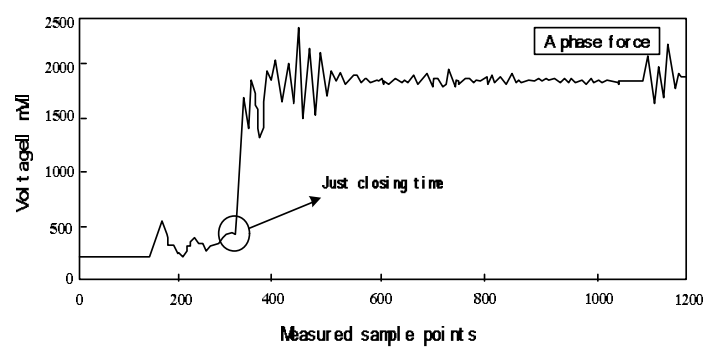

Fig. 3 the measured tension curve of A

The aforementioned data measured curve and simulation curve comparison analysis results show that, the figure in Wavelet analysis is to choose a suitable wavelet base and the corresponding wavelet decomposition level, the closing lever force signal by wavelet packet decomposition and reconstruction, and find out the corresponding just combined moments of characteristic singular point. The closing lever force signal of 5 layers of wavelet packet decomposition and reconstruction of high frequency reconstruction coefficients D5 has a minimum value with just singular points correspond to. Algorithm analysis results are shown in figure 4:

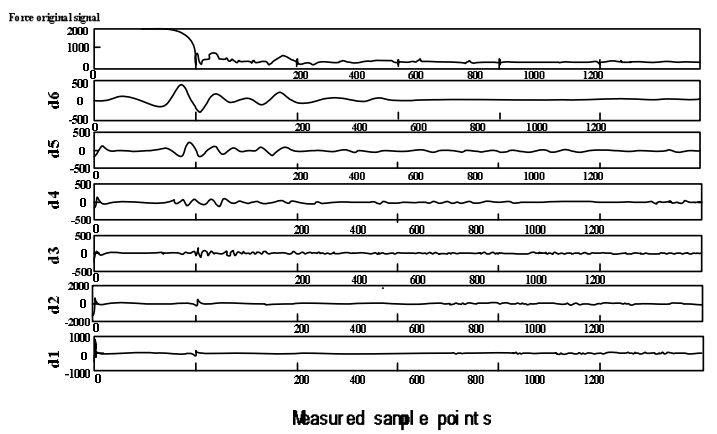

Figure 4 switching force curve Coil2, 5 layer wavelet coefficient reconstruction

The pull force signal in the process of opening and closing of the wavelet packet is decomposed and reconstructed, from fifth layers high frequency reconstruction coefficient D5 minimum, The corresponding time point as just closing time.

\section{Verification of the force signal measurement with just the moment}

Can be seen through the analysis of the above algorithm, Bisection, the process of closing lever force curve decomposition and reconstruction, the extreme of a high frequency coefficient and jus combined point correspondence. So as long as the number of sampling points corresponding to the extreme points, and then combined with the sampling frequency of the test system, Namely: point / sampling frequency, it can be obtained just at the moment.

The starting time of combined operation of the coil electric time is determined, can be obtained by the circuit breaker closing and tripping time. The data measured in the test system is an example, In the main circuit on-off switching time measurement is obtained at the same time, also won the pull force curve, Using wavelet packet decomposition and reconstruction algorithm of curve pull force calculation, switching time parameter can be obtained by force signal measurement. As shown in Figure 5. 


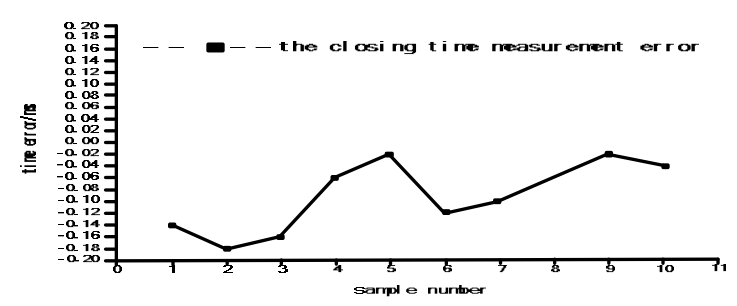

(a) the closing time measurement error

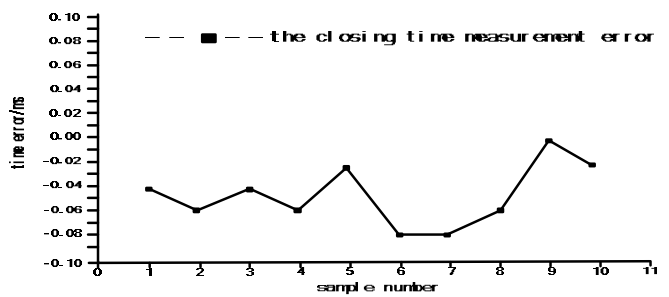

(b) timing measurement error

Figure 5 action time error distribution

By comparative analysis, this paper decomposed and reconstructed the tension force signal produced in the process of opening, closing of circuit breaker by wavelet packet, and made the features of signal more obvious. The measured parameters are compared with the traditional measurement values. The accuracy of the measurement is very high, which indicates that the method is feasible and accurate.

\section{Conclusion}

Circuit breakers work in the environment of strong electromagnetic interference, and lots of interference in the data acquisition exists, thus it needs to select the appropriate algorithm to extract the feature points from the force curve. This paper selected the wavelet packet analysis with time frequency analysis function in the light of the characteristic of existing singular points and the curve of the real measurement force is not stable. The closing rod force signal is decomposed and reconstructed by the six layer decomposition level and a minimum value is corresponding to a singular point for high frequency reconstruction coefficient d6. This paper compared the opening and closing time with traditional measurement values resulted from this method combined with test data of the test system and founded that it has high accuracy.

\section{References}

[1] Chao Fu, Jing Hao. On-line Monitoring System based on Vibration Signal of High Voltage Circuit Breaker[J].Journal of Multimedia, 2014, 9 (4):598-604

[2] Kadri Kadriu, Ali Gashi, Ibrahim Gashi, Ali Hamiti, Gazmend Kabashi. Influence of dc Component during Inadvertent Operation of the High Voltage Generator Circuit Breaker during Mis-Synchronization[J].Energy and Power Engineering, 2013, 05 (03) :225-235

[3] Zhenlu Wang, Xuejin Shen, Xiaoyang Chen. Mechanical Characteristic Analysis of Micro Gear Mechanism [J]. Integrated Ferroelectrics, 2015, 161 (1) : 112-122

[4] V. A. Strunin, L. I. Nikolaeva. Combustion mechanism of RDX and HMX and possibilities of controlling the combustion characteristics of systems based on them[J].Combustion, Explosion, and Shock Waves, 2013, 49 (1) : 53-63 
[5] Liping Dai, Yuhui Ge, Yueming Chen. Research on the Dynamic Mechanism of Characteristics of Upper Echelons[J].iBusiness, 2011, 03 (04),359-365 